\title{
Characterization of $\operatorname{Poly}(N$-isopropylmethacrylamide) in Water
}

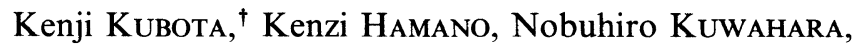 \\ Shouei FujISHIGE, ${ }^{*}$ and Isao ANDo** \\ Department of Biological and Chemical Engineering, Faculty of Engineering, \\ Gunma University, Kiryu, Gunma 376, Japan \\ * Research Institute for Polymers and Textiles, \\ Higashi, Tsukuba 305, Japan \\ **Tokyo Institute of Technology, Ookayama, \\ Meguro-ku, Tokyo 152, Japan
}

(Received May 14, 1990)

\begin{abstract}
Various molecular parameters characterizing solution properties of poly ( $N$-isopropylmethacrylamide) (PNiPMAM) (in the range of moledular weight from 13.0 to $252 \times 10^{4}$ ) in water at $20^{\circ} \mathrm{C}$ were obtained by use of static and dynamic light scattering techniques. The molecular weight dependence of these parameters is expressed by the power law relation and compared with those for poly( $N$-isopropylacrylamide) (PNiPAM) in water. It was found that in aqueous solution PNiPMAM molecules take more expanded structures than PNiPAM molecules due to the presence of methyl group to the $\alpha$-carbon.

KEY WORDS Poly( $N$-isopropylmethacrylamide) / Poly $(N$-isopropylacrylamide) / Light Scattering / Solution Properties / Hydration /
\end{abstract}

Polyacrylamide and its $N$-substituted polymers have attracted much interest due to their unique thermal response in aqueous medium. ${ }^{1}$ For example, aqueous solutions of $\operatorname{poly}(\mathrm{N}$ isopropylacrylamide) (PNiPAM) show that the phase transition takes place reversibly as a function of temperature and almost independently of molecular weight and concentration in the experimental range (concentration lower than $1 \%$ and molecular weight between $5 \times 10^{4}$ and $\left.8.4 \times 10^{6}\right){ }^{2}$ The phase transition temperature of this series of $N$ substituted polyacrylamide derivatives differs strictly depending on the chemical structure of the side chains. ${ }^{3}$ Moreover, gels of polyacrylamide derivatives show a significant volume change with temperature and solvent composition. ${ }^{4,5}$ Especially, the gel of PNiPAM has been used as a standard gel for studying various physicochemical behaviors of aqueous

$\uparrow$ To whom all correspondence should be addressed. gel, ${ }^{6}$ and recently it was shown that the phase transition of this gel can be classified into a three dimensional Ising model system. ${ }^{7}$ This behavior of PNiPAM gels has attracted much interest as a typical functional polymer sensitive to thermal stimulation. Previously, we reported the molecular characterisics of $\mathrm{PNi}$ PAM in water at $20^{\circ} \mathrm{C}$ using well fractionated polymer samples and the relations between the radius of gyration $\left(R_{\mathrm{g}}\right)$, the hydrodynamic radius $\left(R_{\mathrm{h}}\right)$, and the intrinsic viscosity $([\eta])$ and molecular weights were determined. ${ }^{8}$ At $20^{\circ} \mathrm{C}$, the polymer chain behaves as a much hydrated chain and aqueous medium acts as a relatively good solvent thermodynamically. Moreover, it was found that the phase trasition occurs at $31^{\circ} \mathrm{C}$ being accompanied with the coil-globule transition of single polymer chain of PNiPAM molecule in water. ${ }^{9}$ In these analyses, it was clarified that the hydration and hydrophobic interaction play important roles in the thermodynamic behavior of this aqueous 
polymer solution as has been often observed for the non-ionic water soluble polymers. ${ }^{10} \mathrm{~A}$ phase change analogous to the PNiPAM aqueous solutions has been observed for methylcellulose, though the thermal response is not so sharp as that of PNiPAM and is accompanied by a significant retardation for the rate of re-dissolution on cooling. This hysteresis was attributed to the restricted free rotation of the main chain consisting of 1,4- $\beta$-glucoside linkages. This type of thermal response was observed for PNiPMAM aqueous solutions too, ${ }^{2}$ and the phase transition temperature was about $40^{\circ} \mathrm{C}$, higher than that of PNiPAM. Therefore, it is of interest to investigate the effects of the substitution of methyl group to the $\alpha$-carbon of the main chain of PNiPAM and the change of stiffness of polymer chain. Indeed, the effects of methyl group susbstitution to the $\alpha$-carbon have been observed on comparison of solution properties of poly(methylacrylate) to those of poly(methylmethacrylate). ${ }^{11}$ The inverse of parameter $\lambda\left(\lambda^{-1}\right.$ corresponds to the Kuhn's segment length in case of worm-like chain model.) is $2.00 \mathrm{~nm}$ for isotactic poly(methylacrylate) and is $3.27 \mathrm{~nm}$ for isotactic poly(methylmethacrylate). Moreover, because PNiPAM and PNiPMAM molecules have an isopropyl group in every side chain similar to that of valine and leucine of peptide chains, it is also interesting to study the thermodynamic properties of these polymers as a model system for simulating the thermal denaturation of proteins and other biologically important polymers.

In order to study these points in more detail, the overall structure of the polymer chain including polymer dimension, and the relationship of the size of polymer with molecular weight or degree of polymerization were determined by static as well as dynamic light scattering applied for very dilute aqueous polymer solutions.

Here, the power law relationships of static and dynamical polymer size as well as the intrinsic viscosity with the molecular weight and various molecular parameters characterizing PNiPMAM molecules in water at $20^{\circ} \mathrm{C}$ were investigated for comparison with those of PNiPAM.

\section{EXPERIMENTAL}

\section{Materials.}

PNiPMAM samples were polymerized in water by the method of radical polymerization. $N$-Isopropylmethacrylamide was prepared by reaction of isopropylamide with cold methacrylic acid chloride. The monomer was purified by sublimation. It was polymerized in aqueous solution with ammonium persulfate as the initiator at $60^{\circ} \mathrm{C}$ to give high molecular weight polymers. The fractionation of this product was carried out by the phase separation technique in a carefully water-freed acetone $/ n$ hexane mixture at room temperature. This is essentially the same method as was used in the preparation of PNiPAM samples. ${ }^{12}$ Eleven fractions were selected for the present work.

Distilled water of liquid chromatography grade, was purchased from Wako Pure Chemical Industries, and used as supplied.

\section{Intrinsic Viscosity}

Intrinsic viscosities, [ $\eta]$, of all samples used in this study were measured in water at $20.00 \pm 0.05^{\circ} \mathrm{C}$ in an Ubbelohde-type viscometer. The flow times of the sample solutions were long enough so that the kinetic energy correction was negligible.

\section{Light Scattering}

The angular dependences and autocorrelation functions of the scattered light intensity were measured simultaneously by our light scattering apparatus and 240 channels digital correlator. The details have been described elsewhere. ${ }^{13}$ A vertically polarized $488.0 \mathrm{~nm}$ line of Ar-ion laser (NEC, GLG 3200) was used as the incident beam, and vertically polarized scattered light was detected using a photoncounting method. The scattered light intensity 
was observed in an angular range from $20^{\circ}$ to $150^{\circ}$ at $5^{\circ}$ intervals. A cylindrical cell with $10 \mathrm{~mm}$ outer diameter was placed in a silicon oil bath regulated to $20.00 \pm 0.05^{\circ} \mathrm{C}$. Optical clarification of the sample solutions was achieved by direct filtration through a membrane filter, Nucleopore filter. The reduced Rayleigh ratio of the sample solution was calculated using benzene as the standard.

The specific refractive index increment $\mathrm{d} n / \mathrm{d} c$ of PNiPMAM in water at $20^{\circ} \mathrm{C}$ was measured using a Brice type differential refractometer using Ar-ion laser at $488.0 \mathrm{~nm}$, and was $0.162 \mathrm{~cm}^{3} \mathrm{~g}^{-1}$.

From the angular and concentration dependences of the scattered light intensity, the weight averaged molecular weight, $\bar{M}_{w}$, the radius of gyration, $R_{\mathrm{g}}$, and the second virial coefficient, $A_{2}$, were obtained by the usual method. ${ }^{14,15}$

The method of data analysis of the autocorrelation functions of the quasielastically scattered light intensity from the sample solutions were essentially the same to the previous paper. ${ }^{8}$ The hydrodynamic radius, $R_{\mathrm{h}}$, was obtained from the translational diffusion coefficient, $D_{0}$, according to the Stokes-Einstein equation. Third cumulant expansion method was used to obtain the average decay rate or the first cumulant, $\bar{\Gamma}$, as ${ }^{16}$ : $g^{(1)}(\tau)=\exp \left[-\bar{\Gamma} \tau+\left(\mu_{2} / 2 !\right) \tau^{2}-\left(\mu_{3} / 3 !\right) \tau^{3}\right]$

where $g^{(1)}(\tau)$ is the normalized field correlation function, $\tau$ is the delay time and $\mu_{2} / \bar{\Gamma}^{2}$ gives the normalized dispersion of the decay rate distribution which relates to the molecular weight distribution. The concentration dependence coefficient of the translational diffusion coefficient was obtained by

$$
D z(c)=D_{0}\left(1+k_{\mathrm{D}} c+\cdots\right)
$$

where $D z(c)$ is the $z$-averaged translational diffusion coefficient at concentration of $c$ and is determined by the extrapolation of $\bar{\Gamma} / K^{2}$ to $K \rightarrow 0$ with $K$ being the momentum transfer vector.

\section{RESULTS}

One typical example of the Zimm plot for PNiPMAM in water at $20^{\circ} \mathrm{C}$ (sample F) is illustrated in Figure 1, where $I(\theta)$ is the intensity of the light at a scattering angle of $\theta$ and the intensity of depolarized scattered light was negligibly weak and no correction was made. The measured values of $\bar{M}_{w}, A_{2}, R_{\mathrm{g}}$, and $[\eta]$ are summarized in Table I. Figure 2 shows the molecular weight dependence of the second virial coefficient and the following equation was obtained:

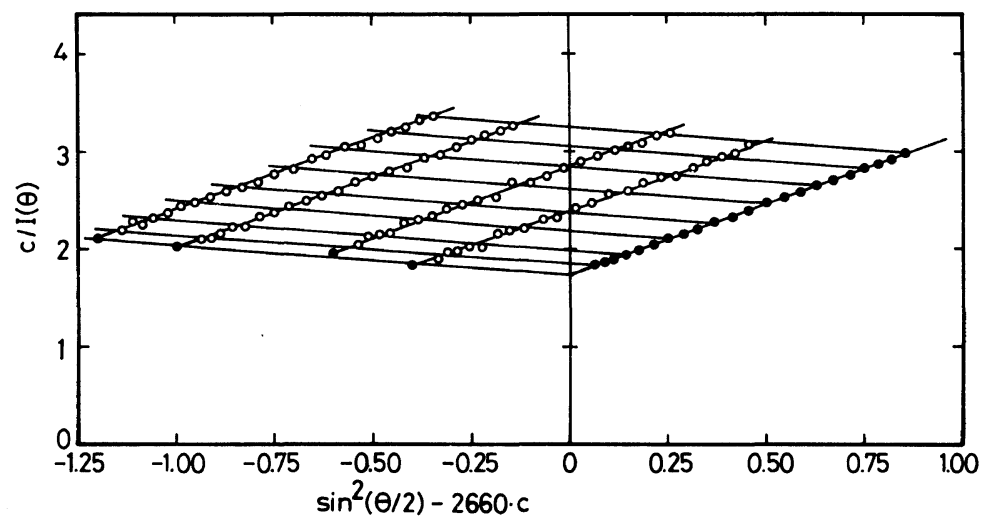

Figure 1. Typical example of Zimm plot of light scattering for poly $(N$-isopropylmethacrylamide $)$ in water at $20^{\circ} \mathrm{C}$. The sample code is $\mathrm{F}$. The unit of $c / I(\theta)$ is arbitrary. 
Table I. Molecular parameters of poly $(N$-isopropylmethacrylamide $)$ in water at $20^{\circ} \mathrm{C}$.

\begin{tabular}{|c|c|c|c|c|c|c|c|c|c|c|}
\hline \multirow{2}{*}{$\begin{array}{l}\text { Sample } \\
\text { code }\end{array}$} & \multirow[t]{2}{*}{$\begin{array}{c}\bar{M}_{w} \\
\times 10^{4}\end{array}$} & \multirow{2}{*}{$\begin{array}{c}\begin{array}{c}A_{2} \\
\times 10^{-4}\end{array} \\
\mathrm{~cm}^{3} \mathrm{~mol} \mathrm{~g}^{-2}\end{array}$} & \multirow{2}{*}{$\frac{R_{\mathrm{g}}}{\mathrm{nm}}$} & \multirow{2}{*}{$\frac{R_{\mathrm{h}}}{\mathrm{nm}}$} & \multirow{2}{*}{$\begin{array}{c}D_{0} \\
\times 10^{-8} \\
\mathrm{~cm}^{2} \mathrm{~s}^{-1}\end{array}$} & \multirow{2}{*}{$\frac{[\eta]}{\mathrm{cm}^{3} \mathrm{~g}^{-1}}$} & \multirow{2}{*}{$\frac{k_{\mathrm{D}}}{\mathrm{cm}^{3} \mathrm{~g}^{-1}}$} & \multirow[t]{2}{*}{$k_{\mathrm{f}, 0}$} & \multirow[t]{2}{*}{$R_{\mathrm{g}} / R_{\mathrm{h}}$} & \multirow[t]{2}{*}{$k_{\mathrm{f}} /[\eta]$} \\
\hline & & & & & & & & & & \\
\hline A & 252 & 1.6 & 76.2 & 50.4 & 4.25 & 178 & 276 & 4.2 & 1.51 & 3.0 \\
\hline B & 187 & 2.1 & 74.5 & 42.0 & 5.11 & 172 & 226 & 5.6 & 1.77 & 3.2 \\
\hline $\mathrm{C}$ & 184 & 1.4 & 67.7 & 39.6 & 5.13 & 162 & 192 & 3.9 & 1.71 & 2.0 \\
\hline D & 124 & 1.7 & 51.1 & 30.5 & 7.03 & 138 & 180 & 4.3 & 1.69 & 1.7 \\
\hline $\mathrm{E}$ & 115 & 2.0 & 55.4 & 30.3 & 7.08 & 126 & 135 & 5.3 & 1.83 & 2.6 \\
\hline $\mathrm{F}$ & 111 & 1.7 & 46.5 & 27.9 & 7.69 & 125 & 141 & 4.8 & 1.67 & 1.9 \\
\hline $\mathrm{G}$ & 99.3 & 1.7 & 46.0 & 27.6 & 7.76 & 112 & 84.6 & 4.6 & 1.67 & 2.2 \\
\hline $\mathrm{H}$ & 94.4 & 1.7 & 46.3 & 28.8 & 7.44 & 108 & 61.7 & 4.1 & 1.61 & 2.4 \\
\hline I & 87.5 & 1.8 & 41.5 & 24.9 & 8.61 & 103 & 94.9 & 4.9 & 1.67 & 2.1 \\
\hline $\mathbf{J}$ & 42.2 & 2.0 & 29.9 & 18.2 & 11.8 & 60 & 19.7 & 4.1 & 1.64 & 2.5 \\
\hline K & 13.0 & 3.6 & 15.0 & 8.4 & 25.5 & 32 & 20.7 & 6.3 & 1.79 & 2.3 \\
\hline
\end{tabular}

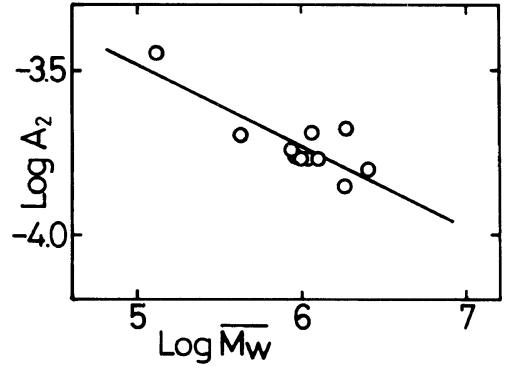

Figure 2. Molecular weight dependence of the second virial coefficient of poly $(N$-isopropylmethacrylamide $)$ in water at $20^{\circ} \mathrm{C}$. The slope of the solid line is -0.25 . The unit of $A_{2}$ is $\mathrm{cm}^{3} \mathrm{~mol} \mathrm{~g}^{-2}$.

$$
A_{2}=5.9 \times 10^{-3} \bar{M}_{w}{ }^{-0.25}\left(\mathrm{~cm}^{3} \mathrm{molg}^{-2}\right)
$$

The molecular weight dependence of the radius of gyration and intrinsic viscosity are shown in Figure 3. Reasonable linear relationship are seen in the double logarithmic plots and the power law relations are obtained by the least squares method:

$$
\begin{gathered}
R_{\mathrm{g}}=1.58 \times 10^{-2} \bar{M}_{w}^{0.58}(\mathrm{~nm}) \\
{[\eta]=9.66 \times 10^{-3} \bar{M}_{w}^{0.68}\left(\mathrm{~cm}^{3} \mathrm{~g}^{-1}\right)}
\end{gathered}
$$

The results of $D_{0}, k_{\mathrm{D}}$, and $R_{\mathrm{h}}$ as well as $k_{\mathrm{f}, 0}$, $k_{\mathrm{f}} /[\eta]$, and $R_{\mathrm{g}} / R_{\mathrm{h}}$ are shown in Table I. $k_{\mathrm{f}, 0}$ is calculated from the equation according to the Pyun and Fixman theory ${ }^{17}$ :

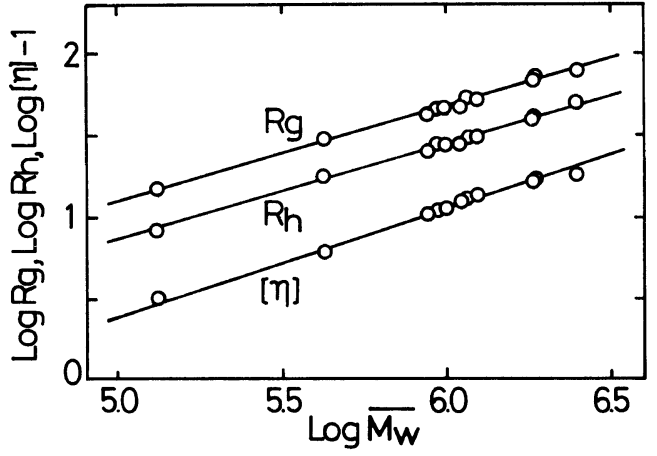

Figure 3. Radius of gyration, $R_{\mathrm{g}}$, hydrodynamic radius, $R_{\mathrm{h}}$, and intrinsic viscosity [ $\left.\eta\right]$, of $\operatorname{poly}(N$-isopropylmethacrylamide) in water at $20^{\circ} \mathrm{C}$ plotted as a function of molecular weight. The slopes of $R_{\mathrm{g}}, R_{\mathrm{h}}$, and [ $\left.\eta\right]$ are 0.58 , 0.57 , and 0.68 , respectively. The unit of $R_{\mathrm{g}}$ and $R_{\mathrm{h}}$ is $\mathrm{nm}$ and that of $[\eta]$ is $\mathrm{cm}^{2} \mathrm{~g}^{-1}$.

$$
\begin{gathered}
k_{\mathrm{f}}=2 A_{2} \bar{M}_{w}-k_{\mathrm{D}}-\bar{v} \\
k_{\mathrm{f}, 0}=k_{\mathrm{f}} \bar{M}_{w} / N_{\mathrm{A}}\left(4 \pi R_{\mathrm{h}}{ }^{3} / 3\right)
\end{gathered}
$$

where $\bar{v}$ is the partial specific volume of the polymer, $N_{\mathrm{A}}$ is Avogadro's constant, and $k_{\mathrm{f}}$ is the concentration dependence coefficient of the friction coefficient, $f(c)$, defined in the following equation:

$$
f(c)=f_{0}\left(1+k_{\mathrm{f}} c+\cdots\right)
$$

The diffusion coefficient plotted against the 


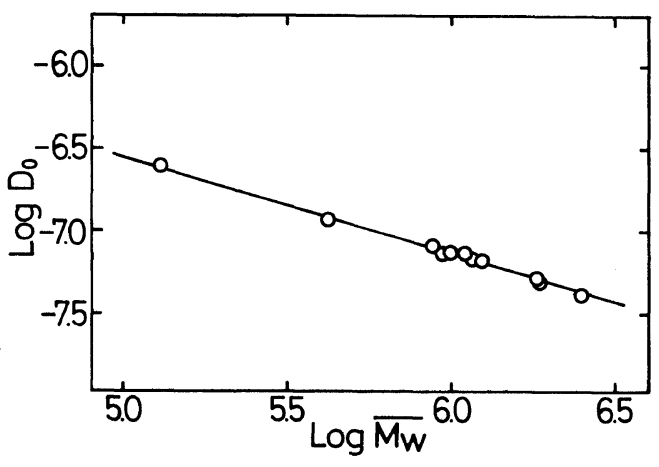

Figure 4. Double-logarithmic plot of the translational diffusion coefficient, $D_{0}$, against molecular weight for poly ( $N$-isopropylmethacrylamide) in water at $20^{\circ} \mathrm{C}$. $D_{0}$ is obrained using the Stokes-Einstein equation from the correlation function measurements. The slope of the solid line is -0.57 . The unit of $D_{0}$ is $\mathrm{cm}^{2} \mathrm{~s}^{-1}$.

molecular weight is shown in Figure 4 and the following equation was obtained:

$$
D_{0}=2.02 \times 10^{-4} \bar{M}_{w}^{-0.57}\left(\mathrm{~cm}^{2} \mathrm{~s}^{-1}\right)
$$

The hydrodynamic radius obtained from the translational diffusion coefficient is shown in Figure 3 and the following equation was obtained:

$$
R_{\mathrm{h}}=1.06 \times 10^{-2} \bar{M}_{w}^{0.57}(\mathrm{~nm})
$$

The typical value of $\mu_{2} / \bar{\Gamma}^{2}$ for PNiPMAM in water was about 0.2 and is very close to our previous results for PNiPAM, the $\bar{M}_{w} / \bar{M}_{n}$ of which were about 1.3 .

\section{DISCUSSION}

The exponents of $R_{\mathrm{g}}\left(v_{\mathrm{g}}\right)$ and $R_{\mathrm{h}}\left(v_{\mathrm{h}}\right)$ against the molecular weight have been discussed experimentally and theoretically. ${ }^{18,19}$ The theoretical value in the asymptotic limit of the excluded volume effect is 0.6 in the Flory's theory in contrast to 0.588 in the renormalization group theory for both exponents. ${ }^{20}$ There seems to remain some controversy about the consistency of $v_{\mathrm{g}}$ and $v_{\mathrm{h}}{ }^{21}$ The present results show that both of $v_{\mathrm{g}}$ and $v_{\mathrm{h}}$ are almost same and very close to the theoretical values. The phase transition temperature is about $40^{\circ} \mathrm{C}$ and

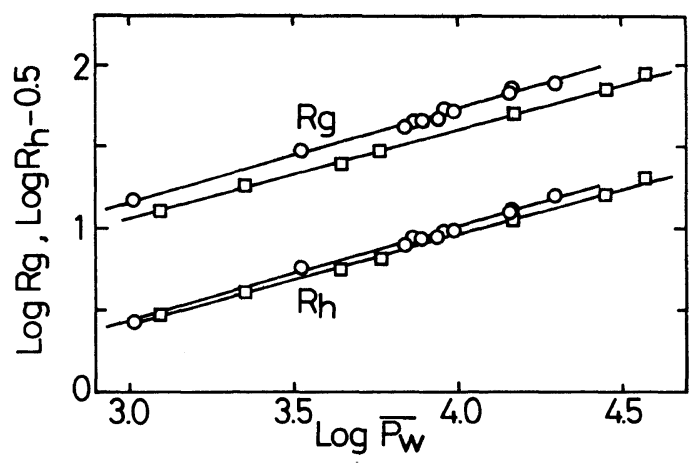

Figure 5. Relationship of radius of gyration, $R_{\mathrm{g}}$, and hydrodynamic radius, $R_{\mathrm{h}}$, with weight averaged degree of polymerization for poly $(N$-isopropylmethacrylamide) (O) and poly $\left(N\right.$-isopropylacrylamide) $(\square)$ in water at $20^{\circ} \mathrm{C}$. The data of poly( $N$-isopropylacrylamide) are from our previous results. The unit of $R_{\mathrm{g}}$ and $R_{\mathrm{h}}$ is $\mathrm{nm}$.

the experimental temperature, $20^{\circ} \mathrm{C}$ is fairly below so that water is considered a sufficiently good solvent in this condition. This is suggested also in the molecular weight dependence of the second virial coefficient. Comparing the exponents with those of PNiPAM $\left(v_{\mathrm{g}}=0.54\right.$ and $\left.v_{\mathrm{h}}=0.54\right)$, the present results are a little larger.

Relationships of the radius of gyration and hydrodynamic radius with the degree of polymerization of PNiPMAM as compared with those of PNiPAM in water at $20^{\circ} \mathrm{C}$ are shown in Figure 5. As is clearly seen, both the radius of gyration and hydrodynamic radius of PNiPMAM are larger than those of PNiPAM. It was confirmed that the increase of the radius of gyration is more sensitive than that of the hydrodynamic radius. This corresponds to the increase of the ratio of $R_{\mathrm{g}} / R_{\mathrm{h}}$ of PNiPMAM compared with that of PNiPAM. The average value of $R_{\mathrm{g}} / R_{\mathrm{h}}$ of PNiPMAM is 1.69 in contrast to 1.38 of PNiPAM. This ratio is related to solvent permeability in the polymer chain and/or chain distribution. ${ }^{22,23}$ For linear chains in good solvents, this ratio has been deduced on the basis of the blob model. ${ }^{24,25}$ Akcasu et al. reported that this ratio should be in a range from 1.5 (at the $\Theta$ condition) to 1.86 (in good solvent). ${ }^{26}$ The present result is in this 
range, though the ratio of PNiPAM is a little lower than that predicted at the $\Theta$ condition. (This lower value observed for PNiPAM aqueous solution might be attributed to the effects of hydration and the resultant increase of effective hydrodynamic interaction.) Therefore, in this respect too, it is suggested that PNiPMAM molecules take more expanded conformation than that PNiPAM molecules.

In order to discuss the intrinsic chain stiffness apart from the excluded volum effect, the direct estimation of the unperturbed chain dimensions is most suitable. However, at the present stage, the $\Theta$ temperature is not yet known. It is expected therefore, that the approximate evaluation of those can be achieved by use of the well founded theoretical frame work of the excluded volume effect, the (modified) Flory-Krigbaum-Orofino theory, ${ }^{23}$ through the interpenetration function, $\Psi$, because the relationships of the second virial coefficients and the radii of gyration with temperature of PNiPAM in water was well predicted by such a theory. The interpenetration function is defined by

$$
\Psi=A_{2} M_{\mathrm{w}}{ }^{2} / 4 \pi^{3 / 2} N_{\mathrm{A}} R_{\mathrm{g}}{ }^{3}
$$

The (modified) Flory-Krigbaum-Orofino theory expresses that

$$
\begin{gathered}
\Psi=\ln \left(1+5.73 Z / \alpha_{\mathrm{s}}{ }^{3}\right) / 5.73 \\
\alpha_{\mathrm{s}}{ }^{5}-\alpha_{\mathrm{s}}{ }^{3}=1.276 Z
\end{gathered}
$$

where $Z$ is the excluded volume function and $\alpha_{s}$ is the expansion factor of the radius of gyration. By this method the unperturbed chain dimension of PNiPMAM was estimated and $R_{\mathrm{g}, 0}{ }^{2} / \bar{M}_{w}=1.7 \times 10^{-17} \mathrm{~cm}^{2} \mathrm{molg}^{-1}$ and $R_{\mathrm{g}, 0}{ }^{2} / \bar{P}_{w}=2.2 \times 10^{-15} \mathrm{~cm}^{1}$ averaging over all samples, $P_{w}$ being the weight averaged degree of polymerization. On the other hand, for PNiPAM $R_{\mathrm{g}, 0}{ }^{2} / \bar{M}_{w}=0.98 \times 10^{-17} \mathrm{~cm}^{2} \mathrm{~mol} \mathrm{~g}{ }^{-1}$ and $R_{\mathrm{g}, 0}{ }^{2} / \bar{P}_{w}=1.1 \times 10^{-15} \mathrm{~cm}^{2} . R_{\mathrm{g}, 0}{ }^{2} / \bar{P}_{w}$ of PNiPMAM is twice as large as that of PNiPAM. This again suggests that the conformation of PNiPMAM is stiffer. Further- more, the characteristic ratio $C_{\infty}$ was estimated, definition of $C_{\infty}$ was described in the previous paper. ${ }^{8}$ The length of monomer unit is related to the shift factor $M_{\mathrm{L}}$, defined by the mass per unit contour length of the polymer chain. ${ }^{11}$ By use of $M_{\mathrm{L}}$ values of isotactic poly(methyl methacrylate) and isotactic poly(methylacrylate), $335 \mathrm{~nm}^{-1}$ and $334 \mathrm{~nm}^{-1}$, respectively, ${ }^{11}$ the characteristic ratio is estimated to be 15 for PNiPMAM and 10 for PNiPAM. If we assume the mean length of monomer unit to be $0.25 \mathrm{~nm}$ as is commonly adopted for vinyl polymers, these are evaluated as 21 for PNiPMAN and 11 for PNiPAM. Both numerical values are comparable with those of flexible linear polymers. This means again that PNiPMAM and PNiPAM are flexible polymers but PNiPMAM is stiffer than PNiPAM. The larger value of $C_{\infty}$ of PNiPMAM compared with PNiPAM results from the effective increase of the restriction of the free rotation due to the change of hydrated structure around the polymer chain originated by the presence of methyl group introduced to $\alpha$-carbon of the repeating unit of the main chain. Because, the difference of the characteristic ratios of polystyrene and poly $(\alpha$-methylstyrene) or poly(methyl acrylate) and poly(methyl methacrylate) is not so large, ${ }^{29}$ and the observed relatively large difference of the characteristic ratios between PNiPMAM and PNiPAM could not result from the restricted free rotation by the steric hindrance alone. The effect of the change of hydrated structure in PNiPMAM is consistent with the higher value of the phase change temperature for PNiPMAN compared with PNiPAM in aqueous solution.

Another important finding is the value of $k_{\mathrm{f}}$ or $k_{\mathrm{f}, 0}$. According to Pyun and Fixman, $k_{\mathrm{f}, 0}$ is a measure of coil interpenetration for a flexible chain and is large as about 7 in the case of low interpenetration and 2.23 for fully interpenetrating coils. ${ }^{17}$ For PNiPAM $k_{\mathrm{f}, 0}$ was 5.3 in water and 3.1 in tetrahydrofuran. It is known that $k_{\mathrm{f}, 0}$ of nonpolar polymer in organic 
solvent is about $3 .{ }^{27}$ In the present case of PNiPMAM in water, $k_{\mathrm{f}, 0}$ was 4.7 and this is very close to the result of PNiPAM in water. This suggests that PNiPMAM molecules might be hydrated extremely as was noted in PNiPAM aqueous solution at low temperatures. The values of $k_{\mathrm{f}} /[\eta]$ seem to be independent of the molecular weight and 2.4 on the average. This is larger than the reported value by Wales et al. for flexible polymers. ${ }^{28}$ But the theoretical treatment of this parameter is not well established, and detailed discussion should be open to future works.

In conclusion, molecular parameters characterizing PNiPMAM in water at $20^{\circ} \mathrm{C}$ were obtained and analyzed in terms of polymer solution theories. It is concluded that PNiPMAM molecules in water at $20^{\circ} \mathrm{C}$ are hydrated flexible coils and the conformation of the polymer chain expands more than PNiPAM molecules and the hydrated structure around the polymer chain should be modified compared with PNiPAM due to the presence of methyl group to $\alpha$-carbon of the repeating unit of the main chain.

Acknowledgements. The authors gratefully acknowledge the assistance of Mr. H. Saitou. This work was partly supported by a Grantin-Aid from the Ministry of Education, Science, and Culture of Japan.

\section{REFERENCES}

1. S. Fujishige and S. Itoh, "Chromic Materials and Its Applications," K. Ichimura, Ed., CMC, Tokyo, 1989.

2. S. Fujishige, K. Kubota, and I. Ando. J. Phys. Chem., 93, 3311 (1989).

3. S. Itoh, Koubunshi Ronbunshu, 46, 427 (1989).

4. T. Tanaka, Phys. Rev. Lett., 40, 427 (1978).
5. T. Tanaka, Sci. Am., 244, 124 (1981).

6. T. Tanaka, "Dynamic Light Scattering," R. Pecora, Ed., Plenum Press, New York, N. Y., 1985.

7. Y. Li and T. Tanaka, J. Chem. Phys., 90, 5161 (1989).

8. K. Kubota, S. Fujishige, and I. Ando, Polym. J., 22, 15 (1990).

9. K. Kubota, S. Fujishige, and I. Ando, J. Phys. Chem., 94, 5154, (1990).

10. W. Burchard, "Chemistry and Technology of Water Soluble Polymers," C. A. Finch, Ed., Plenum Press, New York, N. Y., 1983.

11. H. Yamakawa, Ann. Rev. Phys. Chem., 35, 23 (1984).

12. S. Fujishige, Polym. J., 19, 287 (1987).

13. K. Kubota, H. Urabe, Y. Tominaga, and S. Fujime, Macromolecules, 17, 2096 (1984).

14. M. B. Huglin, "Light Scattering from Polymer Solutions," Academic Press, New York, N.Y., 1972.

15. B. Chu, "Laser Light Scattering," Academic Press, New York, N.Y., 1974.

16. D. E. Koppel, J. Chem. Phys., 57, 4814 (1972).

17. C. W. Pyun and M. Fixman, J. Chem. Phys., 41, 937 (1964).

18. P. J. Flory, "Principles of Polymer Chemistry," Cornell University Press, Ithaca, N.Y., 1953.

19. P. G. de Gennes, "Scaling Concepts in Polymer Physics," Cornell University Press, Ithaca, N.Y., 1979.

20. J. C. Le Guillou and J. Zinn-Justin, Phys. Rev. Lett., 39, 95 (1977).

21. For example, D. W. Schaefer, J. F. Joanny, and P. Pincus, Macromolecules, 13, 1280 (1980).

22. W. Burchard, M. Schmidt, and W. H. Stockmayer, Macromolecules, 13, 1265 (1980).

23. H. Yamakawa, "Modern Theory of Polymer Solutions," Harper and Row, New York, N.Y., 1971.

24. M. Benmouna and A. Z. Akcasu, Macromolecules, 11, 1187 (1978).

25. J. des Cloizeaux and G. Weill, J. Phys. (Paris), 40, 99 (1979).

26. A. Z. Akcasu, M. Benmouna, and S. Alkhafaji, Macromolecules, 14, 147 (1981).

27. K. Kubota, K. M. Abbey, and B. Chu, Macromolecules, 17, 1726 (1984).

28. M. Wales and K. E. van Holde, J. Polym. Sci., 14, 81 (1954).

29. J. Brandrup and E. H. Immergut, Ed., "Polymer Handbook," 3rd ed, John Wiley and Sons, New York, N. Y., 1989. 